\title{
Treatment with a Small Synthetic Compound, KMU-193, induces Apoptosis in A549 Human Lung Carcinoma Cells through p53 Up-Regulation
}

\author{
Eun Young Choi ${ }^{1}$, Kyeong-Cheol Shin ${ }^{1}$, Jinho Lee ${ }^{2}$, Taeg Kyu Kwon ${ }^{3}$,Shin Kim ${ }^{3 *}$, \\ Jong-Wook Park ${ }^{3 *}$
}

\begin{abstract}
Despite recent advances in therapeutic strategies for lung cancer, mortality still is increasing. In the present study, we investigated the anti-cancer effects of KMU-193, 2-(4-Ethoxy-phenyl)-N-\{5-[2-fluoro-4-(4-methylpiperazine-1-carbonyl)-phenylamino]-1H-indazol-3-yl\}-acetamide in a human non-small cell lung cancer cell line A549. KMU-193 strongly inhibited the proliferation of A549 cells, but it did not have anti-proliferative effect in other types of cancer cell lines. KMU-193 further induced apoptosis in association with activation of caspase-3 and cleavage of PLC- $\gamma 1$. However, KMU-193 had no apoptotic effect in untransformed cells such as TMCK-1 and BEAS-2B. Interestingly, pretreatment with z-VAD-fmk, a pan-caspase inhibitor, strongly abrogated KMU193-induced apoptosis. KMU-193 treatment enhanced the expression levels of p53 and PUMA. Importantly, p53 siRNA transfection attenuated KMU-193-induced apoptosis. Collectively, these results for the first time demonstrate that KMU-193 has strong apoptotic effects on A549 cells and these are largely mediated through caspase-3- and p53-dependent pathways.
\end{abstract}

Keywords: KMU-193 - lung cancer - Apoptosis - caspase - p53

Asian Pac J Cancer Prev, 16 (14), 5883-5887

\section{Introduction}

Lung cancer is one of the most frequent malignant tumors in the world and is associated with a high mortality and low cure rates (Jemal et al., 2010; Saika and Machii, 2012; Siegel et al., 2012). Although a multidisciplinary approach has been improved clinical outcomes of patients at early or locally-advanced stages of lung cancer, most patients diagnosed with lung cancer already have advanced disease (Binder and Hegenbarth, 2013). Therefore, systemic therapies are necessary for treatment of lung cancer. The disease control has been achieved with classical doublet chemotherapy in advanced or metastatic non-small cell lung cancer. However it is usually restricted to only a few months (Scagliotti et al., 2002; Schiller et al., 2002; Fossella et al., 2003; Sledge, 2005; Binder and Hegenbarth, 2013). Though, recently, therapies directed against the epidermal growth factor receptor have been incorporated into daily clinical practice, the current 5-year survival rate of lung cancer is approximately $20 \%$ (Xu et al., 2011). Therefore, development of novel therapeutic agents is needed to improve outcomes of lung cancer. BAI (2-[1,1'-biphenyl]-4-yl-N-[5-(1,1-dioxo-1 $\lambda(6)$ isothiazolidin-2-yl)-1H-indazol-3-yl]-acetamide) has been recently developed as a novel cyclin-dependent kinase inhibitor, and reported that BAI inhibits proliferation and induces apoptosis via cytochrome $c$ release, activation of caspases, and inhibition of Akt phophorylation as well as cell cycle arrest in various cancer cells (Lee et al., 2008; Shin et al., 2009; Kim et al., 2013). Moreover, it has been demonstrated that BAI can inhibit tumor necrosis factor- $\alpha$-induced expression of cell adhesion molecules (Hong et al., 2012) and interleukin-1 $\beta$-induced expression of cyclooxygenase-2 in A549 human airway cells (Oh et al., 2010), suggesting its anti-inflammatory activity. Furthermore, there are evidences suggesting that BAI could modulate differentially expressed genes (DEGs) with functions related to many important cellular processes in head and neck cancer cells. Some of these DEGs were DNA methyltransferase 1-associated protein 1 , mitochondrial protein $18 \mathrm{kDa}$, nitric oxide synthase interacting protein, glutamine synthetase, replication initiator 1, and so on (Shin et al., 2009).

Although BAI has strong anti-cancer effect on many types of cancer cells, previous studies have suggested that BAI may possess potential toxicity in vivo because of low cancer cell selectivity (Lee et al., 2008; Shin et al., 2009). Thus, to find out more cancer-selective

${ }^{1}$ Department of Internal Medicine, Yeungnam University College of Medicine, ${ }^{2}$ Department of Chemistry, Keimyung University, ${ }^{3}$ Department of Immunology, School of Medicine, Keimyung University, Daegu, South Korea *For correspondence: j303nih@ dsmc.or.kr,god98005@dsmc.or.kr 
compound than BAI, several derivatives of BAI have been recently synthesized and their anti-cancer activities have been evaluated. Among derivatives of BAI, we found that KMU-193, 2-(4-ethoxy-phenyl)-N-\{5-[2fluoro-4-(4-methyl-piperazine-1-carbonyl)-phenylamino]$1 \mathrm{H}$-indazol-3-yl -acetamide, which is 1,1'-biphenyl group at 3-amino position of indazole is replaced with 4-ethoxyphenyl group in order to reduce the lipophilicity of BAI, has anti-cancer activity. The purpose of this study is for the first time to elucidate the potentials of KMU193 to affect apoptosis and identify the biochemical mechanisms of the anti-cancer effect as well as activation of caspase-3 and p53 tumor suppressor in specifically A549 cells.

\section{Materials and Methods}

Materials. KMU-193 was kindly supplied by Dr. Lee, JH (Keimyung University, Daegu, Korea; Figure 1A). Antibodies specific for phospholipase C- $\gamma 1$ (PLC- $\gamma 1$ ), procaspase-3, p53, and p53 upregulated modulator of apoptosis (PUMA) were purchased from Santa Cruz Biotechnology Inc. (SantaCruz, CA, USA). Antibody specific for cleaved caspase-3 was purchased from Cell Signaling Technology (Danvers, MA, USA). Antibody specific for actin was purchased from Sigma (St. Louis, MO, USA). Pan-caspase inhibitor, z-VAD-fmk (z-VAD) was purchased from Biomol (Plymouth Meeting, PA, USA).

Cell Culture. A549 cells (a human alveolar type II epithelial cell-like adenocarcinoma cell line), SNU449 cells (a human hepatoma cell line) and HCT116 cells (a human colorectal cancer cell line) were maintained at $37^{\circ} \mathrm{C}$ in a humidified atmosphere of $95 \%$ air and $5 \% \mathrm{CO}_{2}$ in RPMI 1640 medium supplemented with $10 \%$ heatedinactivated fetal bovine serum (FBS), $2 \mathrm{mM} \mathrm{L}$-glutamine, $100 \mu \mathrm{g} / \mathrm{mL}$ streptomycin and $100 \mu \mathrm{g} / \mathrm{mL}$ penicillin. Caki cells (a human renal cancer cell line), MDA435S cells (a human breast cancer cell line) and TMCK- 1 cells (a mouse kidney cell line) were maintained at $37^{\circ} \mathrm{C}$ in a humidified atmosphere of $95 \%$ air and $5 \% \mathrm{CO}_{2}$ in Dulbecco's modified Eagle's medium supplemented with $10 \%$ FBS, $20 \mathrm{mM}$ Hepes buffer, and $100 \mu \mathrm{g} / \mathrm{mL}$ gentamicin. BEAS2B cells (a human bronchial epithelial cell line) were maintained at $37^{\circ} \mathrm{C}$ in a humidified atmosphere of $95 \%$ air and 5\% CO2 in keratinocyte-SFM (Gibco, Rockville, MD, USA) supplemented with $10 \%$ FBS, $50 \mu \mathrm{g} / \mathrm{mL}$ bovine pituitary extract, $5 \mathrm{ng} / \mathrm{mL}$ human recombinant epidermal growth factor and $1 \mathrm{X}$ antibiotic antimycotic solution (WELGENE, Daegu, Korea).

Sulforhodamine B (SRB) Assay. After incubation time, adherent cell cultures were fixed in situ by adding $50 \mu \mathrm{L}$ of $50 \%$ (w/v) cold of trichloroacetic acid (Sigma-Aldrich) and incubated for $60 \mathrm{~min}$ at $4^{\circ} \mathrm{C}$. The supernatant was discarded and plates were washed 5 times with deionized water and dried. Fifty $\mu$ L of SRB (Sigma-Aldrich) solution $(0.4 \% \mathrm{w} / \mathrm{v})$ in $1 \%$ acetic acid was added to each well and incubated for $30 \mathrm{~min}$ at room temperature. Plates containing SRB solution were washed 5 times with $1 \%$ acetic acid. Then, plates were air dried and $100 \mu \mathrm{L}$ per well of $10 \mathrm{mM}$ Tris base (pH 10.5) (Sigma-Aldrich) were added and the absorbance of each well was read on an enzyme-linked immunosorbent assay reader at $510 \mathrm{~nm}$. Finally, cell survival was measured as the percentage of absorbance compared with that obtained in control cells (non-treated cells).

Flow Cytometry Analysis. For flow cytometry, approximately $1 \times 10^{6}$ cells were suspended in $100 \mathrm{~mL}$ of PBS, and $200 \mathrm{~mL}$ of $95 \%$ ethanol were added during vortex. The cells were incubated at $4^{\circ} \mathrm{C}$ for $1 \mathrm{~h}$, washed with PBS, and resuspended in $250 \mu \mathrm{L}$ of $1.12 \%$ sodium citrate buffer ( $\mathrm{pH} 8.4$ ) together with $12.5 \mu \mathrm{g}$ of RNase. Incubation was continued at $37^{\circ} \mathrm{C}$ for $30 \mathrm{~min}$. The cellular DNA was then stained by applying $250 \mathrm{~mL}$ of propidium iodide $(50 \mu \mathrm{g} / \mathrm{mL})$ for $30 \mathrm{~min}$ at $37^{\circ} \mathrm{C}$. The stained cells were analyzed by fluorescent activated cell sorting (FACS) on a FACScan flow cytometer (BD pharMingen, CA, USA) for relative DNA content based on red fluorescence.

DNA Fragmentation Assay. After treatment with KMU-193 and/or other drugs, A549 cells were lysed in cell lysis buffer [10 mM Tris (pH 7.4), $150 \mathrm{mM} \mathrm{NaCl}$, $5 \mathrm{mM}$ EDTA, and $0.5 \%$ Triton X-100] for $30 \mathrm{~min}$ on ice. Lysates were vortexed and cleared by centrifugation at $10,000 \mathrm{~g}$ for $20 \mathrm{~min}$. Fragmented DNA in the supernatant was extracted with an equal volume of neutral phenol:chloroform:isoamyl alcohol mixture (25:24:1), and analyzed electrophoretically on $2 \%$ agarose gels containing $0.1 \mu \mathrm{g} / \mathrm{mL}$ ethidium bromide.

Asp-Glu-Val-Asp-ase (DEVDase) Activity Assay. To evaluate DEVDase activity, cell lysates were prepared, and assays were performed in 96-well microtiter plates by incubating $30 \mu \mathrm{g}$ of cell lysates in $200 \mathrm{~mL}$ of reaction buffer (1\% NP-40, 20 mM Tris-HCl, pH 7.5, 137 mM NaCl, 10\% glycerol) containing a caspase substrate (Asp-Glu-ValAsp-chromophore-p-nitroanilide) at $5 \mathrm{mM}$. Lysates were incubated at $37^{\circ} \mathrm{C}$ for $2 \mathrm{~h}$. Thereafter, the absorbance at $405 \mathrm{~nm}$ was measured with a spectrophotometer.

Western Blot Analysis. Cells were washed with cold PBS and lysed on ice in a modified RIPA buffer $(50 \mathrm{mM}$ Tris-HCl, pH 7.4, 1\% NP-40, 0.25\% Na-deoxycholate, 150 $\mathrm{mM} \mathrm{NaCl}, 1 \mathrm{mM} \mathrm{Na} 3 \mathrm{VO} 4$, and $1 \mathrm{mM} \mathrm{NaF}$ ) containing protease inhibitors (100 $\mu \mathrm{M}$ phenylmethylsulfonyl fluoride, $10 \mu \mathrm{g} / \mathrm{mL}$ leupeptin, $10 \mu \mathrm{g} / \mathrm{mL}$ pepstatin, $2 \mathrm{mM}$ EDTA). Cell lysates were centrifuged at $13,000 \mathrm{rpm}$ for 15 min at $4{ }^{\circ} \mathrm{C}$, and the supernatant fractions were collected. Proteins were separated by SDS-PAGE and transferred to an Immobilon-P membrane. Specific proteins were detected using an enhanced chemiluminescence Western blot kit according to the manufacturer's instructions.

Small Interfering RNA (siRNA) Transfection. Small interfering RNA targeting human p53 (p53 siRNA) and green fluorescent protein (control siRNA) were obtained from Santa Cruz Biotechnology Inc. and Bioneer (Daejeon, Korea), respectively. A549 cells were seeded $24 \mathrm{~h}$ before transfection at a concentration achieving $70 \%$ confluence. Cells were transfected with $30 \mathrm{nM}$ of p53 siRNA or control siRNA using Lipofectamine RNAiMAX reagent (Invitrogen, Calsbad, CA, USA) as recommended by the manufacturer's protocol. A master mix was created for each individual condition in order to eliminate pipetting errors and to increase consistency between each well. 


\section{Results}

KMU-193 has a specific and strong anti-proliferative effect on A549 human lung cancer cells

First of all, we investigated the effects of KMU-193 on proliferation of various cancer cell lines, including A549 (lung), SNU449 (liver), HCT116 (colon), Caki (kidney), and MDA435S (breast). Respective cells were treated with or without KMU-193 at the indicated concentrations for $48 \mathrm{~h}$, followed by measurement of cell proliferation by SRB assay. As shown in Figure 1B, treatment with KMU193 for $48 \mathrm{~h}$ inhibited the proliferation of only A549 cells in a dose-dependent manner. However, treatment with KMU-193 at the concentrations tested had no effect on the proliferation of the other cancer cells.

\section{KMU-193 induces morphological change and apoptosis of A549 cells}

To further evaluate anti-cancer effect of KMU-193 on A549 cells, A549 cells were treated with or without KMU-193 for $48 \mathrm{~h}$, followed by measurement of cellular morphology. As shown in Figure 2A, KMU193 treatment resulted in progressive morphological changes of typical apoptosis, including cell shrinkage, rounding, and detachment of the cells from the plate, as observed with light microscopy. In addition, KMU-
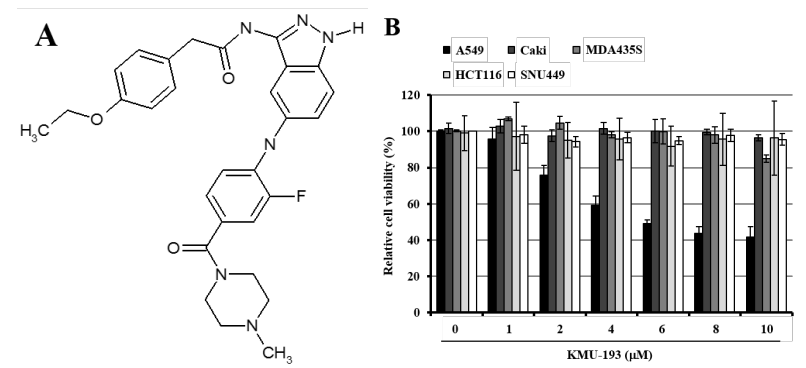

Figure 1. The Structure and Anti-proliferative Effect of KMU-193. (A) The chemical structure of KMU-193. (B) The anti-proliferative effect of KMU-193. A549, Caki, MDA435S, and HCT116 cells were treated with KMU-193 at the indicated concentrations for $48 \mathrm{~h}$. Cell growth was measured by SRB assay. Data are mean values obtained from three independent experiments and bars represent standard deviation
193 induced fragmentation of DNA, a strong index of apoptosis (Figure 2B). It has been reported that only a few chemicals are known to have the property for selective/ preferential elimination of cancer cells without affecting the untransformed cells (Donnerstag et al., 1996; Ahmad et al., 1997). To examine whether KMU-193 triggers cancerspecific apoptosis, two types of untransformed cells were treated with KMU-193 at the indicated concentrations for $36 \mathrm{~h}$. As shown in Figure 2C, the exposure of KMU193 to the two untransformed cells led little changes of cellular viability, however treatment of A549 cells with KMU-193 resulted in markedly increased accumulation of the sub-G1 phase in a dose-dependent manner. These results suggest that KMU-193 may selectively kill lung cancer cells by apoptosis.

Activation of caspases is critical for KMU-193-induced apoptosis of A549 cells

It has been shown that caspase is a key executioner of apoptosis (Logue and Martin, 2008). Therefore, we next investigated whether caspases plays an important role in KMU-193-induced apoptosis. For this, A549 cells were treated with or without KMU-193 and/or z-VAD-fmk, a pan-caspase inhibitor for $36 \mathrm{~h}$, followed measurement of cell cycle by flow cytometry. As shown in Figure 3, pretreatment with z-VAD-fmk strongly abrogated the ability of KMU-193 to increase sub-G1 population in parallel with the decreased levels of pro-caspase-3, the increased levels of cleaved caspase- 3 , to cleave PLC- $\gamma 1$, and to stimulate the DEVDase activity. This finding suggests that KMU-193 induces apoptosis of A549 cells in a caspase-dependent manner.

\section{p53 plays a critical role in KMU-193-induced apoptosis in A549 cells}

The tumor suppressor p53 has been implicated in many important cellular processes, including regulation of apoptotic cell death (Rufini et al., 2013). Evidence suggests crosstalk between p53 and caspases or other apoptosis regulatory proteins in induction of apoptosis in cancer cells (Amaral et al., 2010). Therefore, we next examined 1) whether p53 involves in KMU-193-induced apoptosis in A549 cells and 2) whether KMU-193 regulates
A

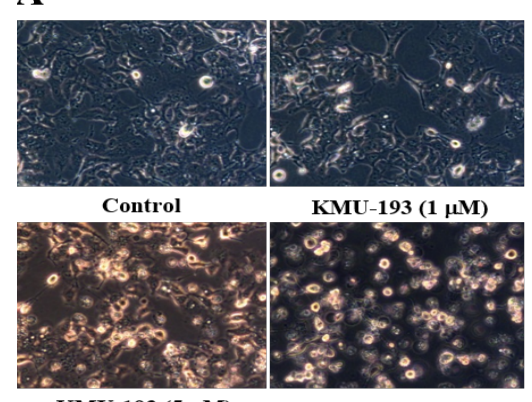

KMU-193 (5 $\mu \mathrm{M})$
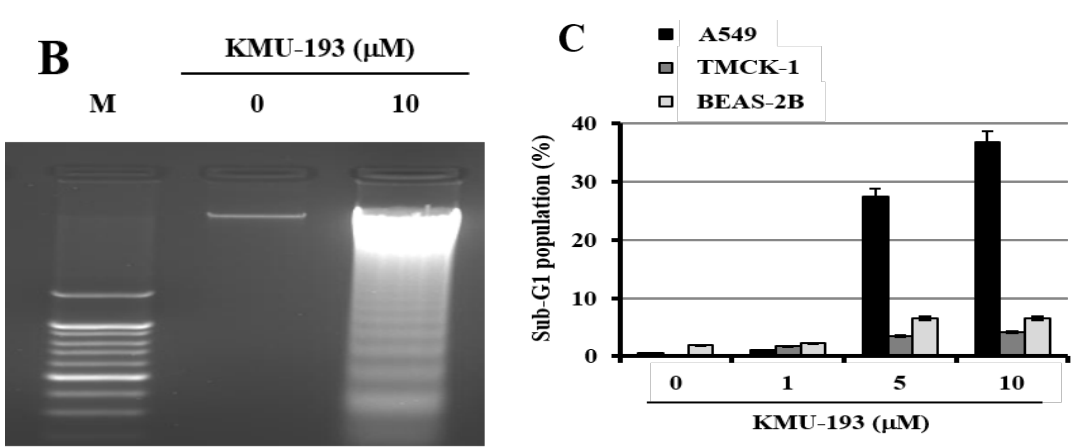

Figure 2. KMU-193 Induces Apoptosis in A549 Cells. A549 Cells were Treated with KMU-193 at the Indicated Concentrations for 36 h. (A) Morphological change was visualized using a light microscopy. (B) Fragmentations of genomic DNA in A549 cells treated with KMU-193. (C) Effect of KMU-193 on growth of untransformed cells. Untransformed TMCK-1 and BEAS-2B cells were treated with the indicated concentrations of KMU-193 for $36 \mathrm{~h}$. The sub-G1 population was measured by flow cytometry as an indicator of the level of apoptosis. Data are mean values obtained from three independent experiments and bars represent standard deviation 

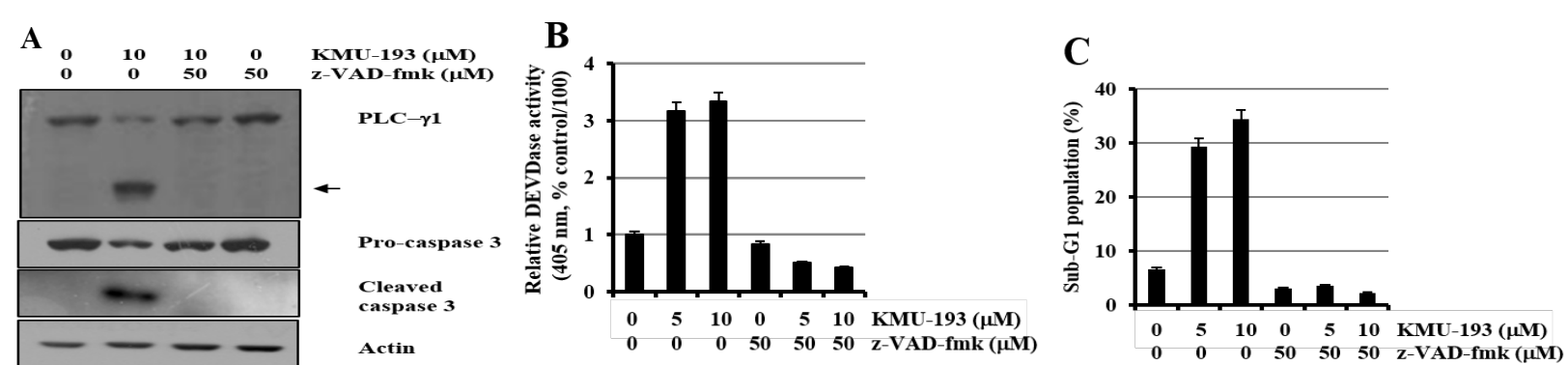

Figure 3. Caspase-dependent Apoptosis in the KMU-193-treated A549 Cells. (A) A549 cells were pretreated with z-VAD-fmk or vehicle for $1 \mathrm{~h}$ then treated with KMU-193 $(5,10 \mu \mathrm{M})$ for $36 \mathrm{~h}$. Whole cell lysates were isolated and analyzed by Western blot with respective antibody. Cleaved form of PLC- $\gamma 1$ is indicated by arrow. Actin was used as a protein loading control. (B) An aliquot of proteins from A was analyzed by an in vitro DEVDase activity using caspase-3 DEVDase assay kits as described in Materials and Methods. (C) The sub-G1 population was measured by flow cytometry as an indicator of the level of apoptosis. Data are mean values obtained from three independent experiments and bars represent standard deviation.
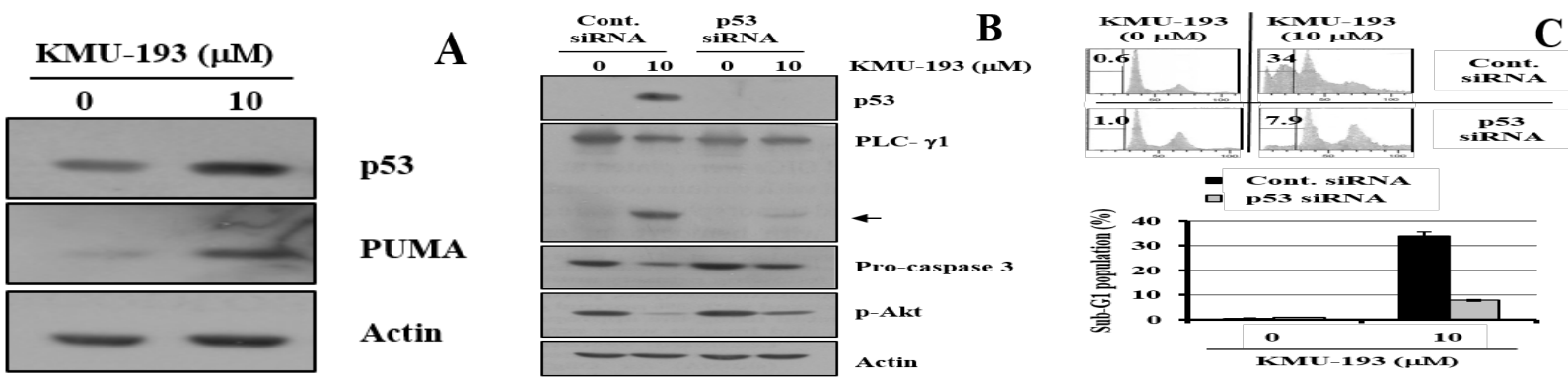

Figure 4. KMU-193-induced p53 Up-regulation is Involved in Apoptosis of A549 Cells. (A) A549 cells were treated with $10 \mu \mathrm{M}$ KMU-193 for $36 \mathrm{~h}$. (B) A549 cells were transiently transfected with control (Cont. siRNA) or p53 siRNA. Thirty hours after transfection, cells were treated with KMU-193 (10 $\mu \mathrm{M})$ for $36 \mathrm{~h}$. Whole cell lysates were isolated and analyzed by Western blot with respective antibody. Cleaved PLC- $\gamma 1$ is indicated by arrow. Actin was used as a protein loading control. (C) Apoptotic cells were determined as the percentage cells in sub-G1 as assessed by flow cytometry. Data are mean values obtained from three independent experiments and bars represent standard deviation

the expression level of p53 and its downstream substrate such as PUMA. The exposure of KMU-193 to A549 cells resulted in markedly increased the expression levels of p53 and PUMA (Figure 4A). To investigate whether the increased expression level of p53 is important to induce apoptosis in KMU-193 treated A549 cells, we employed the siRNA duplex against p53 mRNA. A549 cells were transfected with the p53 siRNA and treated with or without KMU-193 for 36h. Immunoblot analysis demonstrated that transfection of siRNA against $\mathrm{p} 53$ resulted in a suppression of 553 expression in A549 cells as compared to cells transfected with control GFP siRNA (Figure 4B). Under these conditions, the cleavages of PLC- $\gamma 1$ and pro-caspase-3 were blocked by p53 siRNA transfection (Figure 4B). Moreover, the exposure of KMU-193 to p53 siRNA-transfected A549 cells abrogated inactivation of Akt, but not down-regulation of XIAP. As shown in Figure 4C, KMU-193-induced accumulation of the sub-G1 population was inhibited in cells transfected with p53 siRNA as compared to control siRNA-transfected cells. Taken together, these results suggest that up-regulation of p53 may participate in KMU-193-induced apoptosis and inactivation of Akt in A549 cells.

\section{Discussion}

KMU-193, one of BAI derivatives, has been recently developed to improve the selectivity over cancer cell lines. In the present study, we demonstrate for the first time that
KMU-193 has a selective anti-cancer activity against A549 lung cancer cells, which is supported by that KMU-193 does not influence the growth of other types of cancer cells, including SNU449 (liver), HCT116 (colon), Caki (kidney), and MDA435S (breast) and it has no apoptosisinducing effect on BEAS-2B human bronchial epithelial cells and TMCK-1 mouse kidney cells, which may suggest that KMU-193 may be applied as a potential therapeutic agent against lung cancer.

Induction of apoptosis is associated with a variety of proteins and/or factors. Among those, caspase- 3 is one of important cell death-inducing proteases that cleave a number of proteins essential for cell survival (Emoto et al., 1995; Bae et al., 2000). In this study, KMU-193 treatment induced cleavage of pro-caspase- 3 and PLC- $\gamma 1$, activation of DEVDase activity, and apoptosis (DNA fragmentation) in A549 cells. Importantly, we have demonstrated that activation of caspases plays a critical role in KMU-193induced apoptotic pathways in A549 cells, as deduced from that z-VAD-fmk, a pan-caspase inhibitor interferes with KMU-193-induced apoptosis, DEVDase activation, and cleavage of PLC- $\gamma 1$ in the cells.

p53 is the most extensively studied tumor suppressor protein and has been shown to mediate a variety of anti-proliferative process in response to diverse forms of cellular stress (Fridman and Lowe, 2003). Evidence further indicates that PUMA (p53-up-regulated modulator of apoptosis) is a p53-regulating gene that is a key executor of cancer cell death or alive (Nakano and Vousden, 2001). 
Recent studies revealed that apoptosis of lung cancer A549 cells accompanies with p53 up-regulation (Li et al., 2015) and PUMA effectively inhibited cellular proliferation in lung cancer A549 cells (Liu et al., 2015). The present study has revealed that KMU-193 treatment leads to up-regulation of p53 and PUMA in A549 cells, and p53 siRNA transfection markedly reduces KMU-193-induced apoptosis, activation of caspases, and cleavage of PLC- $\gamma 1$ in A549 cells. These findings indicate that p53 also plays an important role in the apoptotic pathway triggered by KMU-193 in A549 cells. Increasing evidence suggests a close link between induction of apoptosis in lung cancer cells and expression and/or activity of apoptosis-regulated protein, including p-Akt (Kim et al., 2013; Wu et al., 2015). Besides, it has been reported that $\mathrm{p} 53$ can activate the PTEN promoter leading to increased expression levels of PTEN, a negative regulator of the PI3K/Akt pathway (Feng, 2010). In the present study, we have shown that p53 siRNA transfection leads to a partial blockage of inactivation of p-Akt in KMU-193-treated A549 cells. These results imply that dephosphorylation of Akt is partially regulated by $\mathrm{p} 53$ and pAkt may play an important role in A549 cell death induced by KMU-193-mediated p53 up-regulation.

In conclusion, we have demonstrated that KMU-193 selectively induces apoptosis in A549 human lung cancer cells and apoptotic effect of KMU-193 on A549 cells is largely mediated via modulation of caspase-3 and p53. Though more in vitro and in vivo studies are needed to establish the anti-tumor activity and mechanisms of a novel BAI derivative, KMU-193, findings presented herein collectively provide fundamental insight of the usefulness of KMU-193 in human lung cancer therapy.

\section{Acknowledgements}

This work was supported by the National Research Foundation of Korea (NRF) Grant funded by the Korean Government (MSIP) (No. 2014R1A5A2010008).

\section{References}

Ahmad N, Feyes DK, Nieminen AL, et al (1997). Green tea constituent epigallocatechin-3-gallate and induction of apoptosis and cell cycle arrest in human carcinoma cells. $J$ Natl Cancer Inst, 89, 1881-6.

Amaral JD, Xavier JM, Steer CJ, et al (2010). The role of p53 in apoptosis. Discov Med, 9, 145-52.

Bae SS, Perry DK, Oh YS, et al (2000). Proteolytic cleavage of phospholipase C-gamma1 during apoptosis in Molt-4 cells. FASEB J, 14, 1083-92.

Binder D, Hegenbarth K (2013). Emerging options for the management of non-small cell lung cancer. Clin Med Insights Oncol, 7, 221-34.

Donnerstag B, Ohlenschlager G, Cinatl J, et al (1996). Reduced glutathione and S-acetylglutathione as selective apoptosisinducing agents in cancer therapy. Cancer Lett, 110, 63-70.

Emoto Y, Manome Y, Meinhardt G, et al (1995). Proteolytic activation of protein kinase $\mathrm{C}$ delta by an ICE-like protease in apoptotic cells. $E M B O J, \mathbf{1 4}, 6148-56$.

Feng Z (2010). p53 regulation of the IGF-1/AKT/mTOR pathways and the endosomal compartment. Cold Spring Harb Perspect Biol, 2, 1057.
Fossella F, Pereira JR, von Pawel J, et al (2003). Randomized, multinational, phase III study of docetaxel plus platinum combinations versus vinorelbine plus cisplatin for advanced non-small-cell lung cancer: the TAX 326 study group. J Clin Oncol, 21, 3016-24.

Fridman JS, Lowe SW (2003). Control of apoptosis by p53. Oncogene, 22, 9030-40.

Hong H, Park YK, Park JW, et al (2012). BAI, a 3-aminoindazole derivative, inhibits interleukin-1beta-induced expression of cyclooxygenase-2 in A549 human airway cells. Int $\mathrm{J} \mathrm{Mol}$ Med, 29, 454-60.

Jemal A, Center MM, DeSantis C, et al (2010). Global patterns of cancer incidence and mortality rates and trends. Cancer Epidemiol Biomarkers Prev, 19, 1893-907.

Kim S, Lee J, Jang BC, et al (2013). BAI, a novel cyclindependent kinase inhibitor induces apoptosis in A549 cells through activation of caspases and inactivation of Akt. J Cell Biochem, 114, 282-93.

Lee J, Choi H, Kim KH, et al (2008). Synthesis and biological evaluation of 3,5-diaminoindazoles as cyclin-dependent kinase inhibitors. Bioorg Med Chem Lett, 18, 2292-5.

Li Q, Ren FQ, Yang CL, et al (2015). Anti-proliferation effects of isorhamnetin on lung cancer cells in vitro and in vivo. Asian Pac J Cancer Prev, 16, 3035-42.

Liu CJ, Zhang XL, Luo DY, et al (2015). Exogenous p53 upregulated modulator of apoptosis (PUMA) decreases growth of lung cancer A549 cells. Asian Pac J Cancer Prev, 16, 741-6.

Logue SE, Martin SJ (2008). Caspase activation cascades in apoptosis. Biochem Soc Trans, 36, 1-9.

Nakano K, Vousden KH (2001). PUMA, a novel proapoptotic gene, is induced by p53. Mol Cell, 7, 683-94.

Oh JH, Park EJ, Park JW, et al (2010). A novel cyclin-dependent kinase inhibitor down-regulates tumor necrosis factor-alpha (TNF-alpha)-induced expression of cell adhesion molecules by inhibition of NF-kappaB activation in human pulmonary epithelial cells. Int Immunopharmacol, 10, 572-9.

Rufini A, Tucci P, Celardo I, et al (2013). Senescence and aging: the critical roles of p53. Oncogene, 32, 5129-43.

Saika K, Machii R (2012). Cancer mortality attributable to tobacco by region based on the WHO Global Report. Jpn J Clin Oncol, 42, 771-2.

Scagliotti GV, De Marinis F, Rinaldi M, et al (2002). Phase III randomized trial comparing three platinum-based doublets in advanced non-small-cell lung cancer. J Clin Oncol, 20, 4285-91.

Schiller JH, Harrington D, Belani CP, et al (2002). Comparison of four chemotherapy regimens for advanced non-small-cell lung cancer. $N$ Engl J Med, 346, 92-8.

Shin HC, Song DW, Baek WK, et al (2009). Anticancer activity and differentially expressed genes in head and neck cancer cells treated with a novel cyclin-dependent kinase inhibitor. Chemotherapy, 55, 353-62.

Siegel R, DeSantis C, Virgo K, et al (2012). Cancer treatment and survivorship statistics, 2012. CA Cancer J Clin, 62, 220-41.

Sledge GW, Jr. (2005). What is targeted therapy? J Clin Oncol, 23, 1614-5.

Wu DM, Zhang P, Xu GC, et al (2015). Pemetrexed induces G1 phase arrest and apoptosis through inhi' biting Akt activation in human non small lung cancer cell line A549. Asian Pac J Cancer Prev, 16, 1507-13.

Xu Y, Zhang Y, Ma S (2011). EGFR inhibitors with concurrent thoracic radiation therapy for locally advanced non-small cell lung cancer. Lung Cancer, 73, 249-55. 